\begin{tabular}{l} 
2. To: (Receiving Organization) \\
DISTRIBUTION \\
\hline 5. Proj./Prog./Dept./Div.: \\
Characterization
\end{tabular}

3. From: (Originating Organization)

8. Originator Remarks:

$$
\text { Fou opporal / Pleaces }
$$

SGN Eurisys Service Corp

6. Design Authority/ Design Agent/Cog.

Engr.:

K.A. White/B.L. Philipp/M.F. Erhart
4. Related EDT No.:

$\mathrm{n} / \mathrm{a}$

7. Purchase Order No.:

9. Equip./Component No.:

$\mathrm{n} / \mathrm{a}$

10. System/BIdg./Facility:

$200 \mathrm{G}$

11. Receiver Remarks: 11A. Design Baseline Document? [x] Yes $\square$ No
12. Major Assm. Dwg. No.:

$\mathrm{n} / \mathrm{a}$

13. Permit/Permit Application No: $\mathrm{n} / \mathbf{a}$

14. Required Response Date:

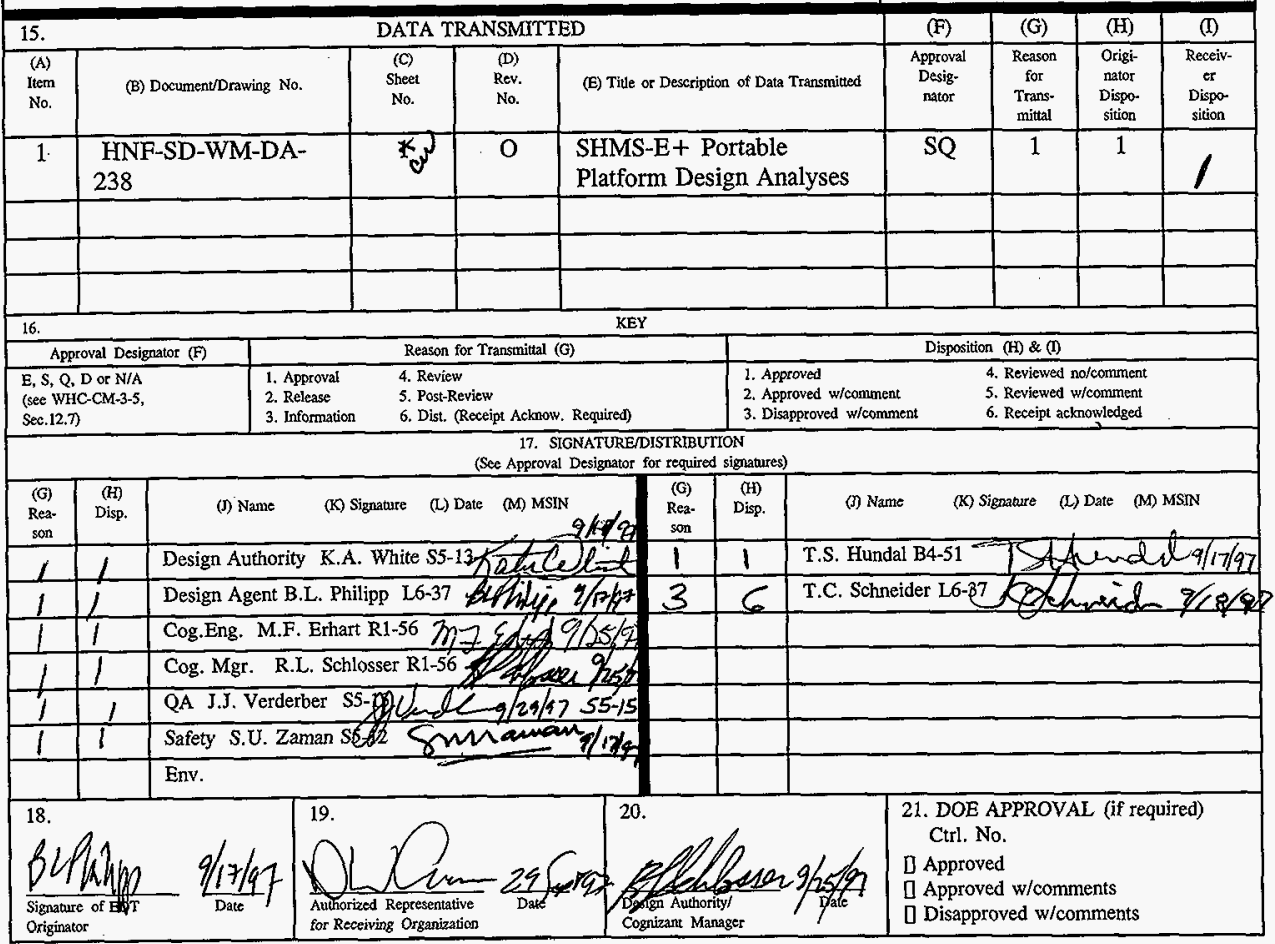




\section{SHMS-E+ PORTABLE PLATFORM DESIGN ANALYSES}

\section{T. S. Hunda1}

SGN Eurisys Services Corporation, Richland. WA 99352

U.S. Department of Energy Contract DE-AC06-96RL13200

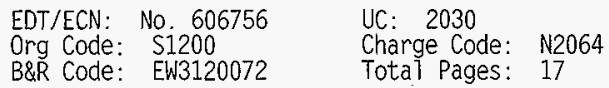

Key Words: Standard Hydrogen Monitoring System (SHMS) Portable Platform Abstract:

Standard-E analytical system design analyses for the Portable Platform.

TRADEMARK DISCLAIMER. Reference herein to any specific commercial product, process, or service by trade name, trademark, manufacturer, or otherwise, does not necessarily constitute or imply its endorsement, recommendation, or favoring by the United States Government or any agency thereof or its contractors or subcontractors.

Printed in the United States of America. To obtain copies of this document, contact: WHC/BCS Document Control Services, P.0. Box 1970, Mailstop H6-08 Richland wA 99352. Phone (509) 372 -2420: Fax (509) 376-4989.
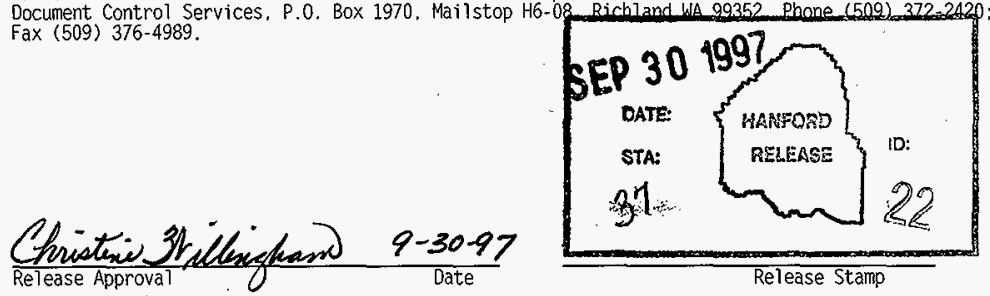

\section{Approved for Public Release}




\section{SHMS-E+ PORTABLE PLATFORM DESIGN ANALYSES}

August 1997

T. S. Hundal

SGN Eurisys Services Corporation 
ANALYTICAL CALCULATIONS

Page 1 of 15

Subject Standard Hvorogen Measurino Svstem (SHMS-E+) Portable Platform Desion

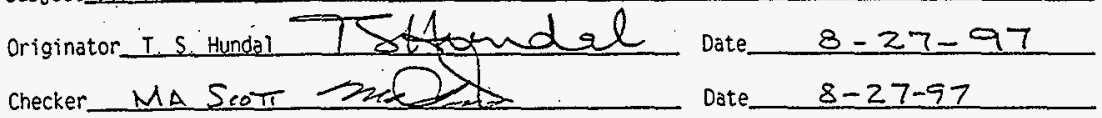

PROBLEM:

Design the portable Standard Hydrogen Monitoring System(SHMS-E+) Platform with installed Bottle Racks. Pump stand. Transformer. Chiller. and SHMS-E test cabinet. This equipment is mounted on the platform. Both Gas Bottle Racks should be designed to withstand seismic loads with adequate anchorage to the platform and the platform itself should be capable of withstanding the applicable wind and seismic loads. The platform with mounted equipment should have lifting lugs to be transported to different test sites within the tank farms.

DESIGN BASIS:

See References on page 2.

ASSUMPTIONS:

All equipment other than the Gas Bottle Racks. mounted on the platform will not fail or collapse when subjected to seismic and wind forces and due to transportation loads.

CONCLUSIONS AND RECOMMENDATIONS:

Use platform details shown on page 3 and as shown on Drawing H-14-102407. SHMS(E+)Platform Assembly. 


\section{ANALYTICAL CALCULATIONS}

\section{Page 2 of 15}

Subject Standard Hydrooen Measuring System (SHMS-E+2 Portable Platform Design

Originator T.S. Hundal

\section{REFERENCES:}

1. Engineering Specifications for Portable Standard Hydrogen Monitoring System(SHMS-E+) Platform. ( Draft Dated 6/10/97).

2. GC-LOAD-01. A/E Civil/Structural Standard Design Criteria. Design Loads for Facilities. (SDC 4.1. Rev. 12)

3. American Institute of Steel Construction. AISC Manual (9th Edition).

4. International Conference of Building Officials Uniform Building Code (UBC). 1991.

5. American Society of Civil Engineers (ASCE) 7-88, Minimum Design Loads for Buildings and other Structures.

6. SM Internationa ?. Metals Handbook (Vol. 1. Tenth Edition).

7. American Welding Society (AWS) D1.1-96. Structural Welding Code-Stee].

8. Quimby \& Associates (Q\&A \# AK9502). Control Skid Structural Calculations

9. DOE-RL-92-36. Hanford Site Hoisting and Rigging Manual. January 15.1993.

10. 101-SY Hydrogen Monitor Gas Bottle Rack. Seismic Analysis. WHC-SD-WM-DA-085, pp 64 thru 78.

11. Seismic/Wind Analysis for Enclosure. WHC-SD-WM-SDD-001. Rev. 0. pp A77 thru A87. (Support Analysis for 101-SY Hydrogen Monitoring System).

12. Drawings: H-14-100837-Sh.1 thru 9, H-2-818214-Sh.1 \& 2, H-14-100844-Sh. 1 thru 9. H-14-100845-Sh.1 \& 2. H-2-85626, H-14-102047 Sh. 1 \& 2.

13. Standard Handbook for Mechanical Engineers, by T. Baumeister and L.S. Marks. Seventh Edition. 
Subject Standard Hydrogen Measurino System (SHMS-E+) Portable Platform Desian

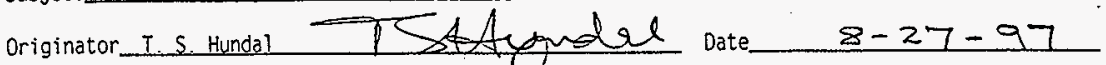
Checker MA Sest Date $8-27-97$

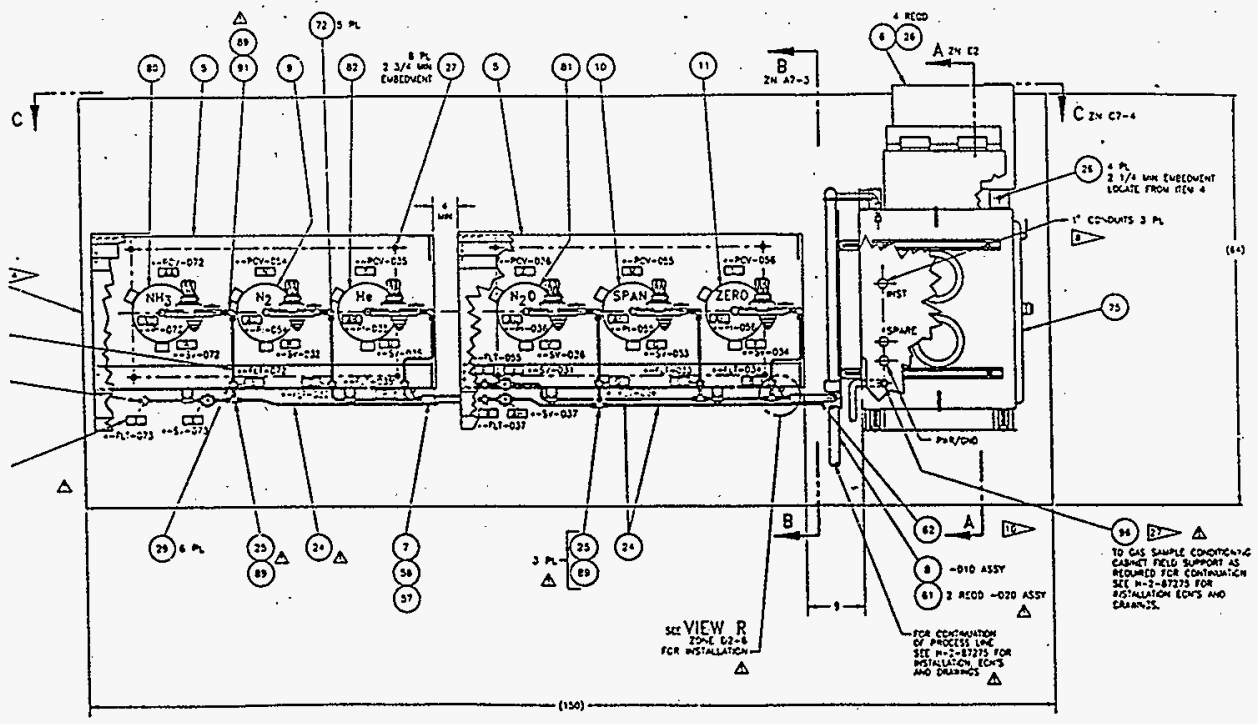

\section{PLAN VIEW}

(1) ANALYTIC EQPT APRANGEMENT DUAL PROCESS LINE

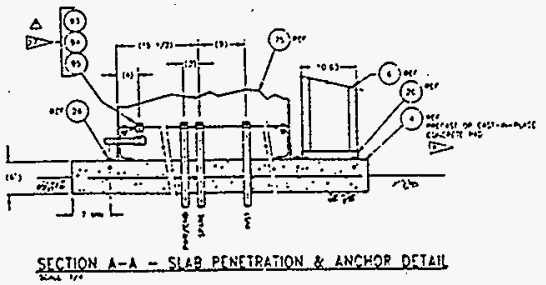

NOTE

CONC. PAD IS FOR REF. ONLY \& TAKEN FROM DWG H-14-100844.

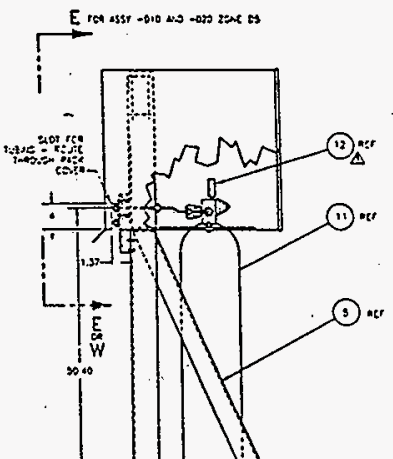


Page_ 5 of 15 ....

Subject Standard Hvdrogen Measuring System (SHMS-E+) Portable Platform Desian '. Originator I.S.Hundal DStuendal checker Ma Seet TH PaC

Date $\quad 8-27-97$

Date $8-27-97$
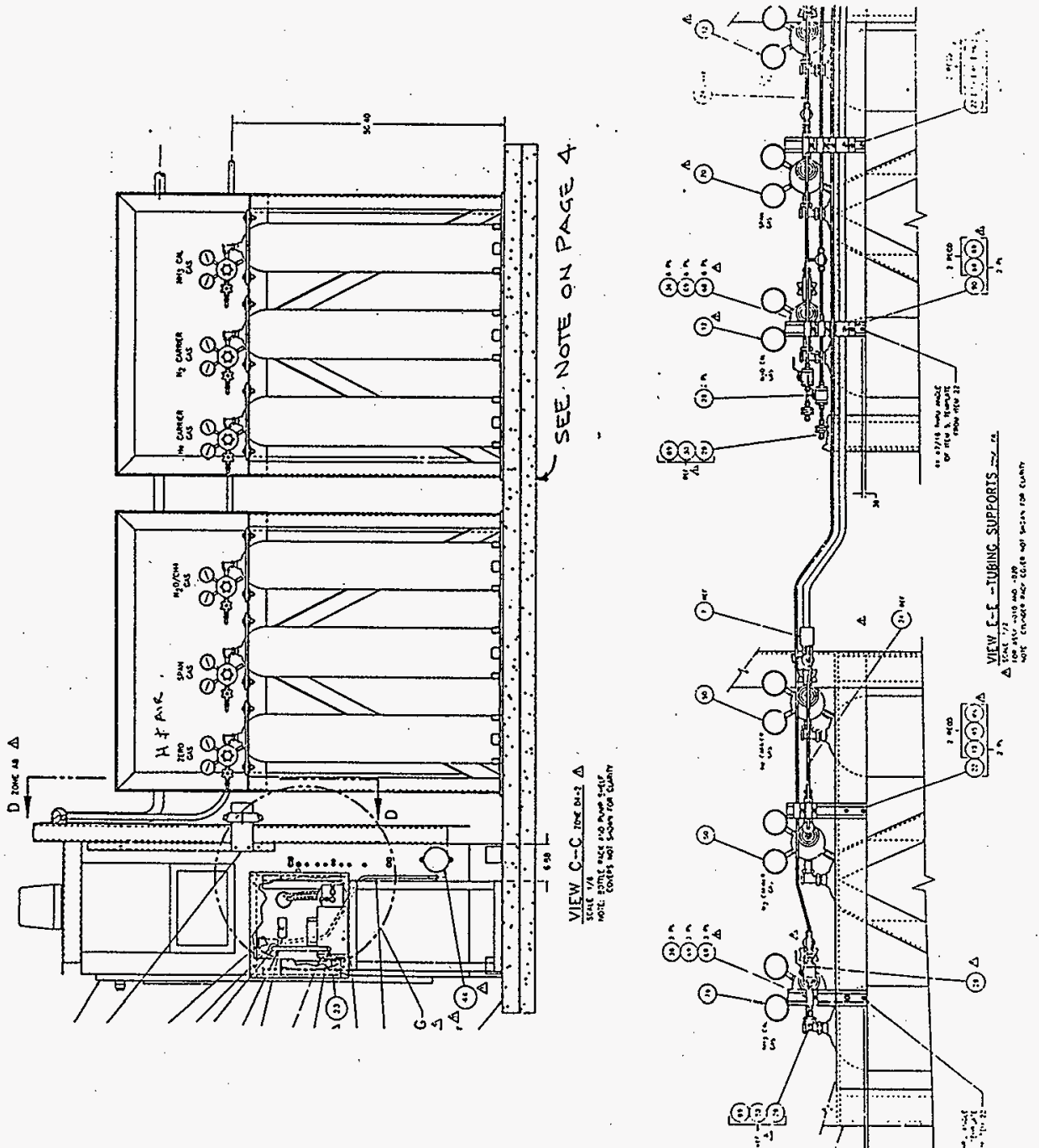
Subject Standard Hydrogen Measuring Svstem (SHMS-E+) Portable Platform Desian originator $T S$ Hundal $M A S c 0 T$ D
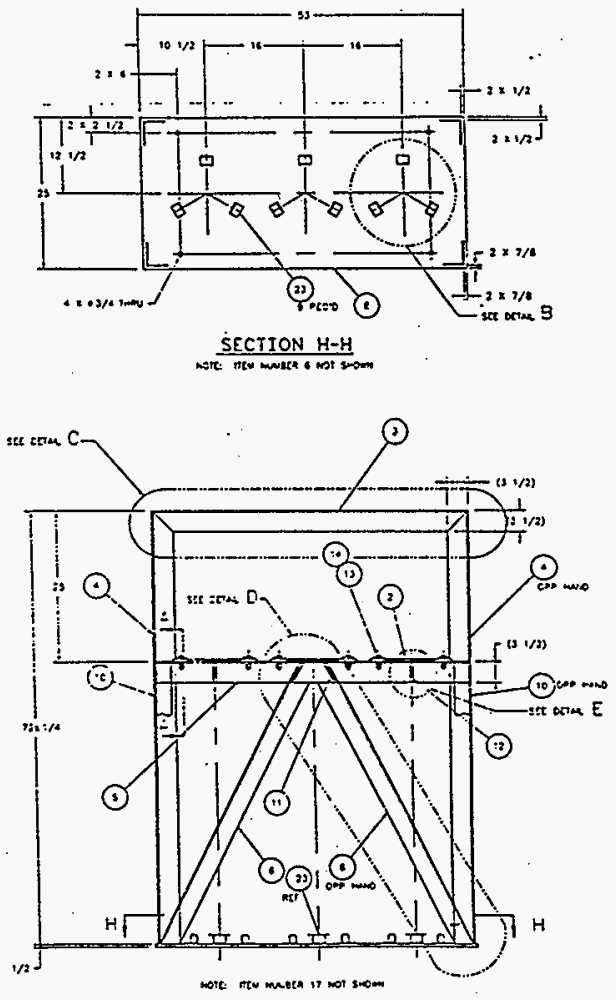

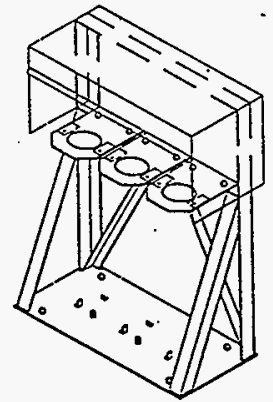

GAS BOTTLE RACK ISONETRIC

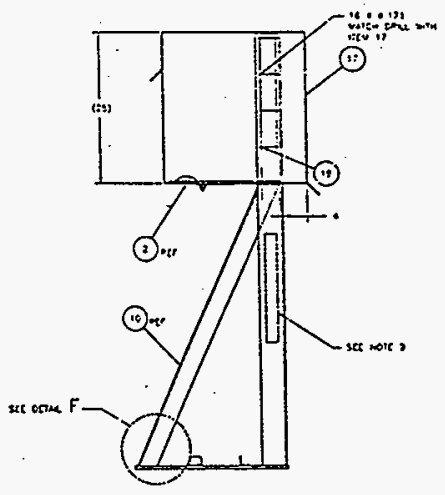

Gas Bottle Rack Detalls 
- analytical calculations

$$
\text { Page } 7 \text { of } 15 \ldots .:=
$$

Subject Standard Hydrooen Measuring System (SHMS-E+) Portable Platform Desion

Originator T.S. Hundal TSLAuadal

Date

$8-27-97$

Checker MASeoT

Date

$8-27-97$
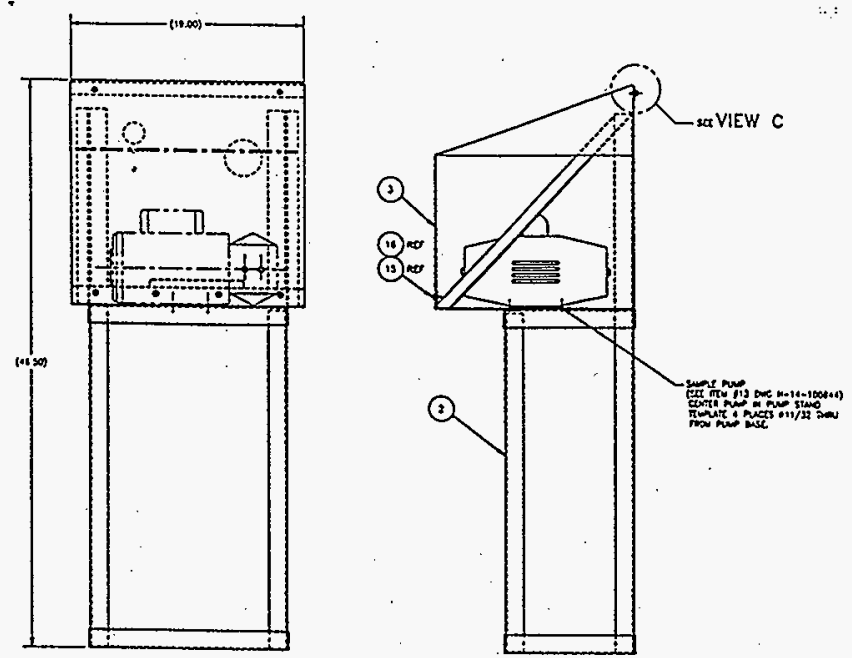

(1) SAMPLE PUMP STAND ASSEMBLYY
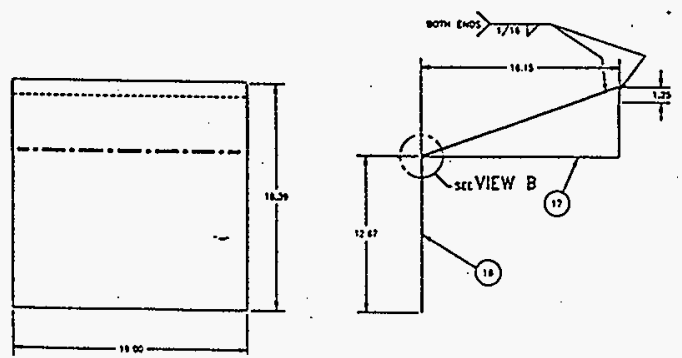
Subject SHMS $(E+)$ PORTAELE PLATFORM

Originator

Checker TStandal $M \triangle S 60 \pi$

TOTAL ASSEMBLED MEGHT OF THE SYSTEM IS 255 LBS.

DOOR SWING is 36 NCHES.

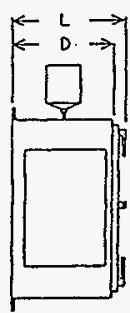

LT. SDE VEH
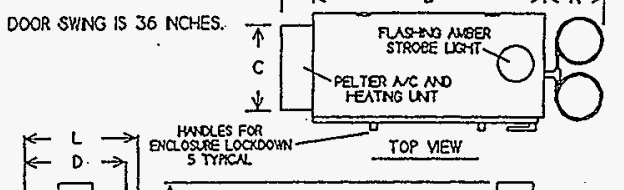

FRONT VEW
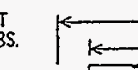

$\sim \wedge$ 2uaDP

\section{in}

$\longrightarrow+N \rightarrow$

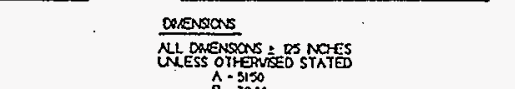

Date $8-27-97$

Date $8-27-97$

NLL DAENGENS D D NCHES

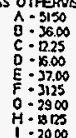

$\rightarrow$ J

$K=0 . \infty$

$\stackrel{L}{\mu}=\frac{10.75}{B .00}$

$N \cdot 1000$

$\stackrel{K}{\underline{y}}$

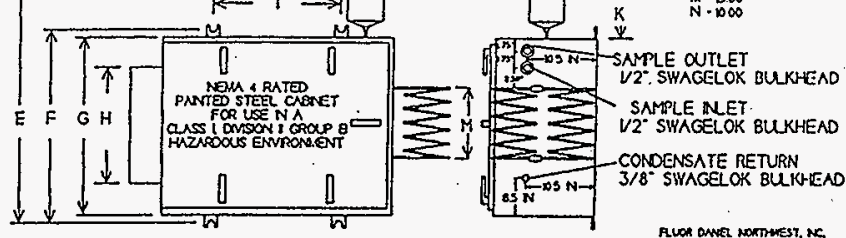

RT. SLE VEY

RLOK ONW NorTHEST, NC.

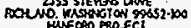

20. $10.0 \times+10+500-10528$
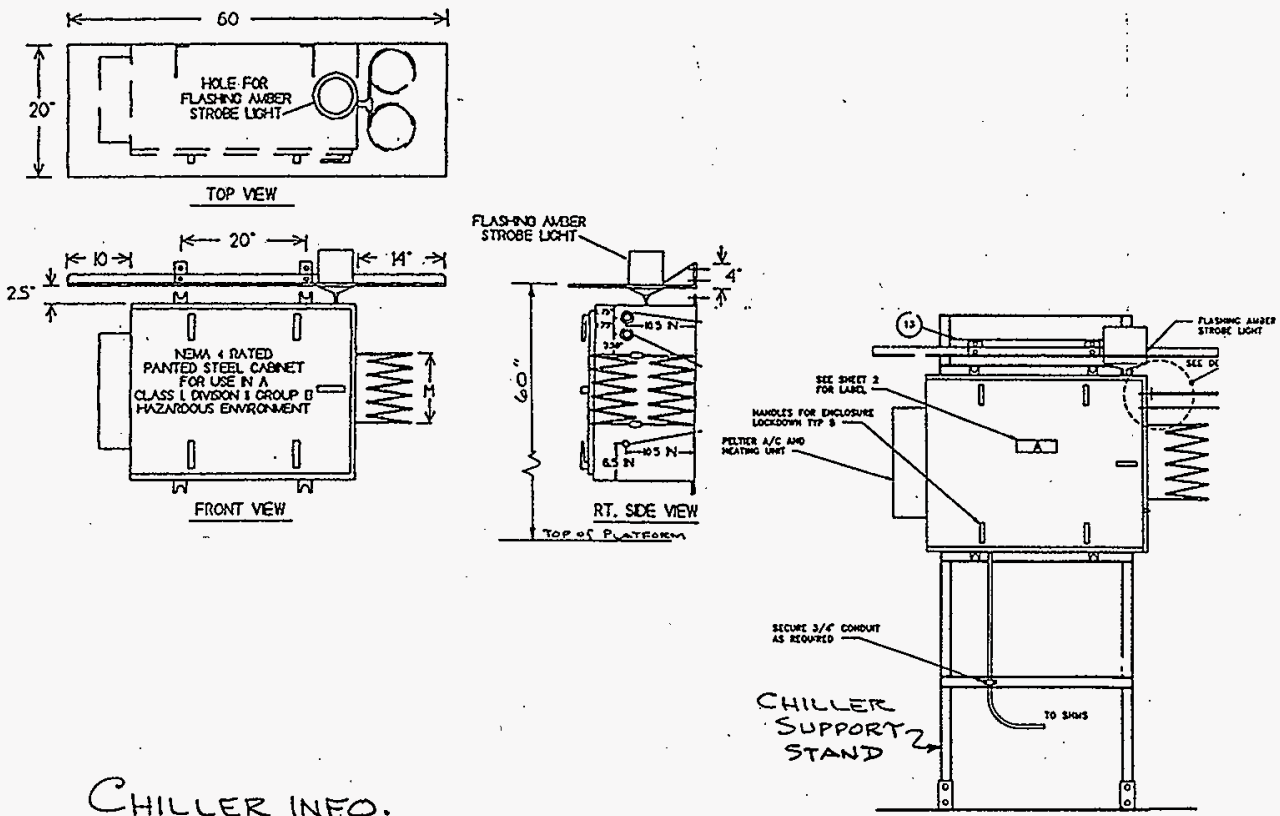

Bhiller INFO. 


\section{ANALYTICAL CALCULATIONS}

Subject SHMS (EH) PORTABLE PLATFÖRM

Page 9 of $15:$

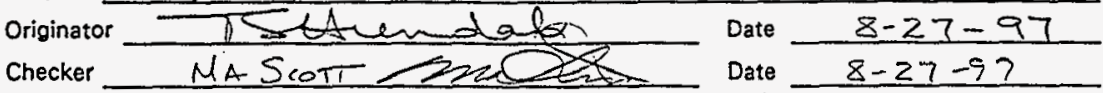

EHECK CGX10.5 $\quad l=14.5^{\prime}, S=5.06, I=15.2$

(EXIST. DWG.H-Z-85626 SH.2)

TOTAL LOADS ON PLATFORM

DL (ESTIMATE)

SHMS CABINET $=900 \%$

ZBOTTLE RACKS W/BOTTLES $=2 \times 630=1260$

PUMP \& STAND= $=100$

CHILLER \& STAND. $. \therefore \quad=250$

TRANSFORMER (EST)......... $300^{\circ}$

PLATFORM 15PSF (EST): $=1100$

MISC. $\quad " \cdot=500^{-}$

TOTAL DL $=4410 \neq$

$\underline{L}$

$25 p=f=25 \times 14.5 \times 5=1820 *$

TOTAL DLTLL $=6230 \#$

DYNAMIC LOADS

DURING TRANSFORTATION, $50 \%=31.5$ \#

(ASSUME):

$\therefore$ TOTAL DESILN LOAD $=9345 \%$

$\therefore$ LOAD/LONC. $C G=\frac{9345}{2} \times 1.15=5.4 \mathrm{k}>5.2^{k} \therefore N G$

TRY C $8 \times 11.5$ * $15 \%$ FOR ASSUMED ASYMMETRY P. $2-87$

ALLOW. LOAD $=8.4^{k}>5.4^{k}:$ ERK

REF 3, p. $2-85$

Us: $\quad$ C $8 \times 11.5$ 
HNF-SD-WM-DA-238, Rev O

Page 11 of 16

ANALYTICAL CALCULATIONS

Page 10 of 15.

Subject SHMS $(E+)$ PORTABLE PLATFORM

Originator

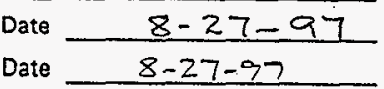

Checker

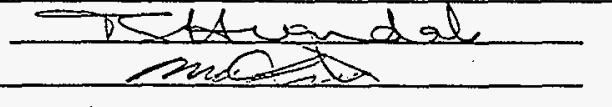

SEISMIC:

CHECK GAS BOTTLE RACK FOR SEISMIC LOADS

(REF. 10), (

SIMS SYSTEM IS GENERAL SERVICE (SCR) (REFT) BUT IT COULD INTERACT WITH SCI SSE.

$\therefore$ IT IS A $4 / 1$ SITUATION

USE REF. 2 \{USEHECMETHOD WITH SCI SEISMIC DEMAND\} ~

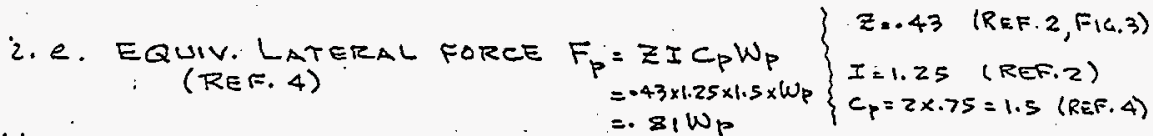

USE REF. 10 RESULTS TO MODIFY THE FINAL FORCES \& stRESES AS FOLLOWS:

MODIFICATION FACTOR, $M_{f}=\frac{.81}{.5}=1.62$

$$
\begin{aligned}
& \frac{24 \times 3 \frac{1}{2} \times x^{3} 8}{f_{a}=14 \times 1.62}=23 \mathrm{pm} \quad f_{v y}=7 \times 1.62=11, \mathrm{ps} \\
& f_{v z}=7 \times 1.62=11 \text { bye } \\
& f_{b x}=108 \times 1.6 z=175 \mathrm{phi} \quad f_{b y}=110 \times 1.6 z=178 \mathrm{pri} \\
& f_{b z}=230 \times 1.62=373 \mathrm{pm} \\
& \angle 3_{2}^{\prime} \times 3^{\prime} 2 \times 5^{\prime} 16 \\
& f_{a}=100 \times 1.62=162 \text { phi } \quad f_{v y}=6 \times 1.62=10 \text { pei } \\
& f_{b x}=53 \times 1.62=86 \text { part } \\
& f_{6 y}=107 \times 1.62=173 p s i \\
& f_{V_{z}}=31 \times 1.62=50 \text { pei } \\
& f_{b z}=994 \times 1.6 z=1610 \mathrm{pr} \\
& \leq 3 \times 3 \times 14 \\
& f_{a}=46 \times 1.6 z=75 \text { per } \quad f_{v y}=9 \times 1.6 z=15 \text { prim } \quad f_{v z}=4 \times 1.6 z=7 \text { prim } \\
& f_{b x}=154 \times 1.62=249 \text { psi } f_{b y}=324 \times 1.62=525 \text { phi } f_{b z}=304 \times 1.6 z=492 \mathrm{por}
\end{aligned}
$$

$1 / 2$ R

$$
\begin{aligned}
& \sigma_{x}=382 \times 1.6 z=619 \text { psi } \quad T_{y}=441 \times 1.6 z=714 \text { psi } \tau_{x y}=97 \times 1.6 z=157 \text { psi } \\
& \sigma_{1}=513 \times 1.6 z=831 p \mathrm{si} \quad \sigma_{z}=509 \times 1.62=8251 \mathrm{psi} \\
& \text { VON MISE s }=448 \times 1.6 z=726 \text { psi }
\end{aligned}
$$

NOTE: BY INSPECTION, STRESSES ARE WELL WITHIN ALLOWABLE LIMITS.

$$
\frac{\text { REACTIONS }}{F_{x}=28.5 \times 1.62=46^{*}}, F_{y}=26.2 \times 1.62=42^{\#}, F_{z}=203.8 \times 1.62=330^{*} \text { USE } 5_{8}^{\prime \prime} \phi \text { A } 307 \text { BOLTS }
$$


HNF-SD-WM-DA-238, Rev O

Page 12 of 16

ANALYTICAL CALCULATIONS

Page 11 of $15 \ldots$ :

Subject SHMS (E+) PORTABLE PLATFORM

Originator

Date

$8-27-97$

Checker

Date

$8-27-97$

WT. Computations (ACTUAL)

PLATFORM FRAMING

$$
\begin{aligned}
& 2-C 8 \times 11.5 \quad(14.5+5) 11.5 \times 2=449 \\
& 2 . c 6 \times 10.5 \quad 15.3 \times 10.5 \times 2=322 \\
& 9-c 6 \times 10.5 \quad 4.625 \times 10.5 \times 9=437 \\
& 1-756 \times 4 x^{3} 16 \quad 4.625 \times 11.97=55 \\
& =2000 \# \\
& 1-R^{1} 14.5^{\prime} \times 5^{\prime} \times 4^{\prime \prime} \\
& 14.5 \times 5 \times 10.2 \\
& =740 \\
& =\overline{2003}
\end{aligned}
$$

GAS BOTTLE RACK W/BOTTLES

$$
\begin{aligned}
& \text { FRAME } \\
& \mathbb{R} 53 \times 25 x^{6} 2 \\
& 2 .<4 \times 3^{1} 2 x^{3} 8 \\
& 1-<4 x^{3} \frac{1}{2} \times 38 \\
& \text { 4. }<3 \times 3 x^{\prime} 4 \\
& 1 \text { - } 3 \frac{1}{2} \times 3 \frac{1}{2} \times 516 \\
& 3-R^{5} 14^{11} \times 20^{11} \times 38 \\
& 9-\angle 1^{\prime} 2 \times 1_{2}^{\prime} \times 3 \times 16 \times 2
\end{aligned}
$$$$
\frac{53 \times 25}{144} \times 20.4=188
$$$$
2 \times 6^{\prime} \times 9.1^{\prime}
$$$$
=109
$$$$
33^{11} / 12 \times 9.1^{1}
$$$$
=40
$$$$
4 \times 4.33^{\prime} \times 4.9^{\prime}
$$$$
=85 .
$$$$
53 / 12 \times 7.2^{1}
$$$$
=32
$$$$
\frac{3 \times 14 \times 20}{144} \times 15.3=89
$$$$
9 \times \frac{2}{12} \times 1.8
$$$$
=3
$$

Ia GaGe GaL. COVER SHT.

$$
\begin{aligned}
\text { IA GALE GAL. COVER SHY. } & \\
\{[(23+6+3)+(25+3)] 53+2 \times 25 \times 23\} \frac{3.75}{144} & =113 \\
\text { BOLTS +MISC } & =10 \\
3 \text { BOTTLES/RACK } 3 \times 122 & =667 . \\
& =366 \\
& \text { Say } 370
\end{aligned}
$$

RACK \# BOTTLES WT., $670+370=1040$ \#

* full bottle

BOTTLE WT. $=115^{*}+7$ CONTENTS 
HNF-SD-WM-DA-238; Rev O

Page 13 of 16

ANALYTICAL CALCULATIONS Page 12 of 15::

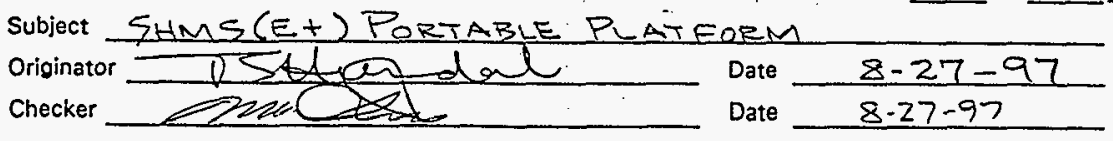

REVISED LOADS ON PLATFORM (DI)

SHMO CABINET

Z-BOTTLE RACKS

PUMP \& STAND

CHILLER \& STAND

TRANSFORMER

PLATFORM

Misc.
$=900 \%$

$2 \times 1040=2080$

$=.100$

$=250$

$=300$

$=2000$

$=100$

TOTAL TL $\because=5730 \#$

LL @25psf $=25 \times 14.5 \times 5:=1820$ \#

DYNAMIC LOADS

DURING TRANSPORTATION, (ASSUME $50 \%$.PL) $=2865$ *

$\therefore$ DESIGN LOAD $=5730+2865=8595^{\circ}$

Assume LOAd on Two LIFT POINTS $=\frac{8595}{2}=4298^{\#}$

ASSUME $15 \%$ ASYMMETRY

$\because\left\{\begin{array}{r}\text { REF. } 9 \\ \text { SEC. } 9.2 .1 .2\end{array}\right\}$

$\therefore$ MAX.LOAD@A LIFT POINT =4298 1.15

$=4942$ Say $5000 \#$

CHECK ACTUAL ASYMMETRY (SEE P.14)

@ $\times$ AXis

$Y=\frac{100 \times 18-(900 \times 6+300 \times 20)}{5730}=1.68^{\prime \prime}$ SAY $Z^{\prime \prime}$

Qr AXIS

$$
\begin{aligned}
& X=\frac{2000 \times 87+250 \times 10+1040(53.5+110.5)+300 \times 128+900 \times 159+100 \times 163}{5730}=95^{\prime \prime} \\
& \therefore \text { ASYMMETRY }=95-87=8^{\prime \prime} \Rightarrow 9.2 \% \quad<15 \% \text { ASSUMED . W }
\end{aligned}
$$


HNF-SD-WM-DA-238, Rev O

Page 14 of 16

ANALYTICAL CALCULATIONS

Subject SHMS(E +) PORTABLE PLATFORM

Originator

Checker

Date

Page 13 of 15

Date $8-27-97$

$8-27-97$

LIFTING LUG CONN.

SPREADER

Hook

HSE A SPREADER BEAM w/ SLING ANGLE $=60^{\circ}$ (MIN.)

$A_{\omega}=2(6+7.25+2)=30.5$ in $\quad \begin{aligned} & S_{x}=6 \times 8+2 \times 7.25+\frac{7.25^{2}}{3} \\ & =80 \mathrm{in}^{2}\end{aligned}$

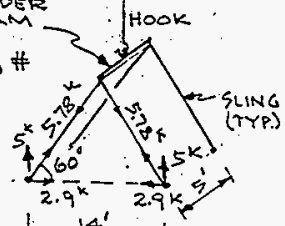

$$
\left.x=\frac{2.5 \times 7.25-(1 \times 7.25+2 \times 2 \times 2)}{2(7.25+2)}=.16^{4}\right\}=80 \mathrm{im}^{2} \text {. }
$$

$J_{\omega}=\frac{6^{3}+3 \times 6 \times 8^{2}}{6}+\frac{7.25^{3}+3 \times 7.25 \times 3.5^{2}}{6}+\frac{2^{3}+3 \times 2 \times 7.25^{2}}{6}=389.8 \mathrm{in}^{3}$

$f_{x}=\frac{2.9}{30.5}+\frac{2.9 \times 6(4)}{389.8}=.27 \mathrm{k} / \mathrm{ing}, f_{z}^{*}=\frac{2.9}{30.5}+\frac{2.9 \times 6}{80}$

$f_{y}=\frac{5}{30.5}+\frac{5 \times 16 \times 3.16}{389.8}=.17 \mathrm{k} / \mathrm{im}$

$$
=.31 \mathrm{k} / \mathrm{im}
$$

*ASSUME NO AM

$$
f_{r}=\sqrt{(27)^{2}+(.17)^{2}+(31)^{2}}=64^{k} / \mathrm{in}<3.6^{k} / \mathrm{hr}
$$

$\rightarrow$ Q

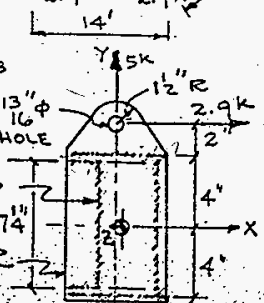

$\left|z_{1}\right| t_{2} \mid$

USE $3_{4}$ "Q SHACKLES W/3 TON MINICAPACTY " WELD CAPACITY

CHECK R

$14.4 \times 25=3.6 k / 1$ in

$$
\begin{aligned}
& \text { Allow. BEARINC LOAD }=-9 F_{Y} A_{P} \\
& f_{b}=\frac{2.9 \times 2}{6 \times(.75) 2 / 6}=10.3 \mathrm{ksi}<24 \mathrm{kh} \quad=.9 \times 36 \times(.75 \times .75) \\
& \text { REF. 3, J8, p.5-79 } \\
& \therefore \text { or } \\
& =18.2^{k}>5.78^{k} \quad \text {. ok }
\end{aligned}
$$

CROSS BEAM $(C 6 \times 10.5)$ :

$$
l=S^{\prime} \quad \text { ALLOW. HDL }=15^{k} \quad \because \text { OK bY INSPECTION }
$$

CHECK OVERTURNING DUE TO SEISMIC LOADS

LATERAL SEISMIC LOAD $=.81 \mathrm{~g} .$.

FOR FLEXIBLE EQUIP. UNGROUND

$$
\begin{aligned}
& =81 \times 5730 \\
& =4641 \#
\end{aligned}
$$

$$
\begin{aligned}
\text { C. } C_{1} & =\frac{2000 \times 6.3+2(670 \times 36+370 \times 28)+900 \times 48+100 \times 56+250(28+16)+300 \times 14+100 \times 38}{5.730} \\
& =26.07^{\prime \prime} \\
\text { F.O.S. } & =\frac{.85 \times 5730 \times 28^{\prime \prime}}{4641 \times 26.07}=1.12>1.0 \quad \text {. OC }
\end{aligned}
$$
SLIDING: SINCE NO CREDIT CAN RE TAKEN FOR FRICTION FOR SEISMIC 
HNF-SD-WM-DA-238, Rev O

Page 15 of 16

ANALYTICAL CALCULATIONS

Subject SHMS (Et) PORTABLE PILAFORM

Originator

Checker

$$
\text { Date } \frac{8-27-97}{8-27-97}
$$

EHËCK PlatforizM against Wind SAFetY

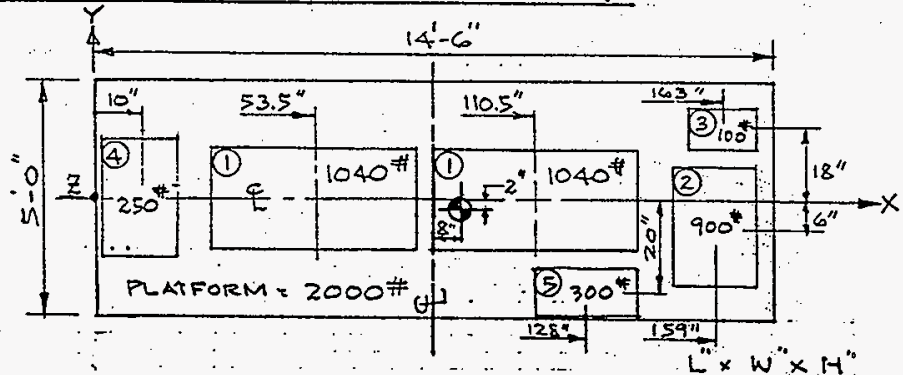

WIND SPEED

(1)-GASBDTTLERACK $53 \times 25 \times 72$

$\mathrm{V}=80$ soph

(2) SMMS CABINET

$32 \times 24 \times 96(72+6+18)$

IMPORTANCE FACTOR $I=1.0$

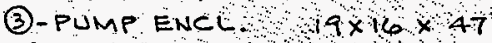

EXPOSURE CLASS = C

(4)- CHILLER

$52 \times 19 \times 60$

USE REF. 5 (ASCE 7-88)

(5). TRANSFCRMER $20 \times 16 \times 27$

DESIGN WIND FORCE, $F=q_{z} G_{h} C_{f} A_{f}$

$$
\begin{aligned}
\text { where: } q & =00256 \mathrm{~K}(\text { IV })^{2} \\
& =.002566 .8)(1 \times 80)^{2} \\
& =13.1 \mathrm{psf}
\end{aligned}
$$

WIND LOAD IN T-DIRECTION

$$
\begin{aligned}
& A_{f_{1}}=\left[\frac{53 \times 72}{144} \times 75\right] 2=40.5 F \\
& A_{f_{2}}=\frac{24 \times 96}{144}=16 \mathrm{sF} \\
& A_{f_{3}}=\frac{19 \times 47 \times * 5}{144}=3 \mathrm{sF} \\
& A_{f_{4}}=\frac{19 \times 60 \times .5}{144}=4 \mathrm{sF} \\
& A_{f_{5}}=\frac{20 \times 27}{140}=4 \mathrm{sF}
\end{aligned}
$$$$
G_{h}=1.32 \text { (TABLE \&) }
$$$$
\therefore c_{f}=1.4 \quad \text { (TABLE 12) }
$$$$
A_{f}=\text { PROJECTED AREA }
$$$$
\text { (c) } 47^{\prime \prime},\left(7 z^{\prime \prime}-25^{\prime \prime}\right) \text { SEEP. }
$$$$
\text { @ 48" }
$$

(c) 38",

$\left(47-9^{\prime \prime}\right)$ SEe $p \cdot 7$

e $44^{\prime \prime},\left\{60-\left(\frac{29+3)}{2}\right\}\right.$

SEE P. 8

$@ 14^{\prime \prime}$ * Approx. SuRfACE AREA 
HNF-SD-WM-DA-238, Rev $O$

Page 16 of 16

ANALYTICAL CALCULATIONS

Page 15 of 15.

Subject SHMS (E+) PORTABLE PLATFORM

Originator

Checker Date $8-27-97$ Date $8-27-97$

$$
F=13.1 \times 1.32 \times 1.4 \times 67=1622 \%
$$

C.G. of PROJECTED AREAS

$$
z=\frac{2(20 \times 4.7)+16 \times 48+3 \times 38+4 \times 44+4 \times 14}{20+20+16+3+4+4}=\frac{3014}{67}=45^{\prime \prime}
$$

CHECK OVERTURNING

ASSUME CG. OF PLATFORM WITH EQUIP. MOUNTED IS ' 6 " OFF \&. (NOTE: 2 "ACTUAL, SEE P.12 \&14)

$$
\begin{array}{r}
\text { F.0.5. }=\frac{5730 \times 24}{1622 \times 53}=1.60>1.0: \text {. ok } \\
\text { (REF. 4 SECT.2317) }
\end{array}
$$

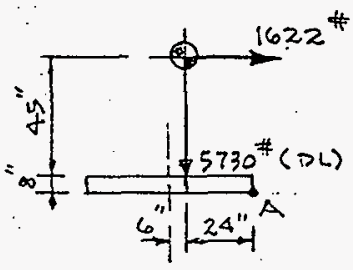

CHECK SLIDING

COEFFICIENT OF FRICTION, $\mu=0.4$ $(\cdot 3$ to $\cdot 7)$

SLIDING FORCE, $S_{f}=16.22$ \# IRON" ON' STONE REF.13 P. 3.38

RESISTING FORCE," $R_{f}=: 4 \times 5730=2292$ \#

$$
\text { F.O.S }=\frac{2292}{1622}=1.41>1.0: \text { Sk }
$$




\section{DISTRIBUTION SHEET}

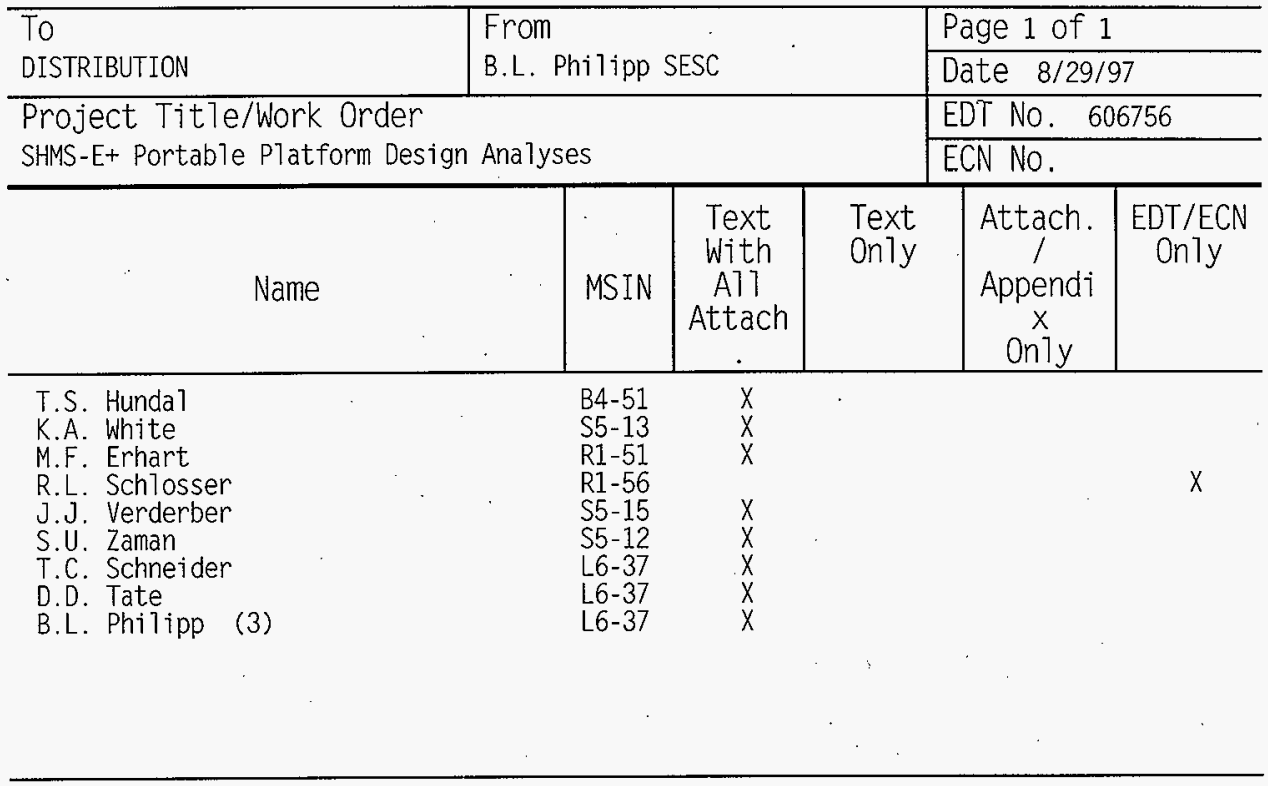

